\title{
Evaluación del método del tubo para concentrar plaquetas caninas: estudio celular
}

\author{
Evaluation of the tube method for concentrating canine platelets: cellular study \\ RF Silva ${ }^{a}{ }^{\text {, }}$, CMF Rezende ${ }^{b}$, FO Paes-Leme ${ }^{b}$, JU Carmona $^{a}$ \\ ${ }^{a}$ Grupo de Investigación Terapia Regenerativa, Departamento de Salud Animal, \\ Universidad de Caldas, Manizales, Caldas, Colombia.

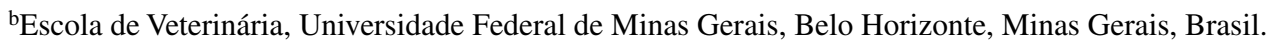

\begin{abstract}
SUMMARY
The aim of this study was to evaluate a manual method for concentrating canine platelets and consequently produce autologous platelet concentrates (APCs) for clinical and experimental proposals. The APCs were obtained by venous blood collection in tubes with ACD solution and were spun at $191 \mathrm{~g}$ for 6 minutes. The cell counts of whole blood and APCs, were statistically different $(\mathrm{P}<0.01)$ between the values for platelets, leukocytes, granulocytes, monocytes, but not for lymphocytes. A strong negative correlation $(\rho=-0.702 \mathrm{P}=0.011)$ between the number of lymphocytes and platelets in the APCs was found. The collection efficiency of platelets was $29.9 \%$ and the concentration of platelets was $49.4 \%$ higher in APCs when compared to the whole blood samples. The results indicated that this simple centrifugation method allows concentrating canine platelets.
\end{abstract}

Palabras clave: perro, plasma rico en plaquetas autólogo, medicina regenerativa, método del tubo.

Key words: dog, autologous platelet rich plasma, regenerative medicine, tube method.

\section{INTRODUCCIÓN}

Los concentrados autólogos de plaquetas (APCs) son fuente de diversos factores de crecimiento (GFs), entre los que se destacan el GF transformante beta I (TGF- $\left.\beta_{1}\right)$, GF insulínico tipo I (IGF-I), GF derivado de las plaquetas (PDGF) y otras moléculas que modulan la inflamación y la reparación tisular (Anitua y col 2004, Argüelles y col 2006). Los APCs han sido ampliamente usados en medicina humana en procedimientos tales como la reconstrucción alveolo-maxilar (Thor y col 2007), y su uso se ha extendido a la cirugía plástica, cirugía ortopédica, medicina deportiva (tratamiento de lesiones tendinosas en atletas), tratamiento de úlceras gástricas, úlceras cutáneas crónicas y úlceras corneales (Anitua y col 2007). En medicina veterinaria se ha reportado la utilización clínica de APCs en equinos para tratar afecciones locomotoras crónicas (Carmona y col 2009, b) y heridas de las extremidades (Monteiro y col 2009), entre otras.

A pesar del uso creciente de APCs en medicina equina (Carmona y Prades 2009), existen escasos reportes sobre el uso clínico de APCs en caninos. Aunque esta especie ha sido ampliamente usada como modelo experimental para evaluar el efecto de esta sustancia como coadyuvante (único o combinado con biomateriales) en la regeneración ósea

\footnotetext{
Aceptado: 08.09.2010.

* Calle 65, No 26-10, Manizales, Caldas, Colombia; raul.silva@ ucaldas.edu.co
}

maxilar (Moreno y col 2004, Bonomi y col 2007, Dutra y col 2008) o apendicular (You y col 2007) y en pruebas de osteointegración (Casati y col 2007). Cabe resaltar que los resultados experimentales obtenidos en estos estudios no han sido muy satisfactorios. Esto debido a la gran disimilitud metodológica y a la falta de una estandarización experimental para elegir el anticoagulante ideal y los parámetros de centrifugación que permitan concentrar un adecuado número de plaquetas en esa especie.

Hasta el momento se han descrito dos métodos para obtener APCs a partir de sangre canina, el método del tubo (manual) (Jensen y col 2004, Choi y col 2005) y el método semiatomatizado SmartPReP 2 system (Thoesen y col 2006). Los autores evaluaron el método descrito para concentrar plaquetas mediante el método del tubo (Jensen y col 2004, Choi y col 2005) y no encontraron reproducibilidad alguna (datos sin publicar) al seguir las recomendaciones de esos investigadores. Por otra parte, el uso de dispositivos semiatomatizados (Thoesen y col 2006) para concentrar plaquetas caninas son costoprohibitivos en la mayoría de los países del continente. El objetivo fundamental de la presente investigación fue estandarizar un método manual que permitiera concentrar plaquetas caninas de manera reproducible con la finalidad de poder usar APCs tanto en el campo clínico como experimental en caninos. La hipótesis del estudio fue que las plaquetas de perro podrían ser concentradas mediante la recolección de sangre entera en tubos con ácido cítrico-citrato de sodio-dextrosa (ACD) centrifugados de manera única. 


\section{MATERIAL Y MÉTODOS}

Este estudio fue aprobado por el comité de ética en experimentación animal de la Universidad Federal de Minas Gerais, Brasil, protocolo número 125/2009.

\section{ANIMALES}

Se utilizaron doce perros de raza Fila Brasilero, seis machos con edades entre 24 y 108 meses y seis hembras con edades entre 18 y 60 meses, clínicamente sanos al momento de la recolección de la sangre y serológicamente negativos a leishmaniosis y erliquiosis.

\section{OBTENCIÓN DEL CONCENTRADO AUTÓLOGO DE PLAQUETAS}

Se realizó extracción de sangre mediante punción de la vena safena con un catéter mariposa $21 \mathrm{G}$ (Shandong Weigao Group, China). La sangre de cada animal fue depositada en un tubo de $10 \mathrm{~mL}$ con 1,5 $\mathrm{mL}$ de solución A de ACD, citrato de trisodio (22 g/L), ácido cítrico (8 g/L) y dextrosa (24,5 g/L) (BD, New Jersey, USA). Una muestra adicional de sangre fue depositada en un tubo de $5 \mathrm{~mL}$ con $\mathrm{k}_{3}$ EDTA para realizar un hemograma. La sangre del tubo con ACD fue centrifugada (SIGMA 3K30, Alemania) a $191 \mathrm{~g}$ durante 6 minutos. Posteriormente, con la ayuda de una micropipeta de volumen fijo de $1000 \mu \mathrm{L}$ se recolectaron (aproximadamente) los primeros $100 \mu \mathrm{L}$ de la porción roja y los primeros $900 \mu \mathrm{L}$ de plasma, por debajo y por encima de la interfaz eritrocitos-plasma, respectivamente. Los APCs obtenidos fueron analizados mediante hemograma automatizado por impedancia volumétrica (Abacus Junior Vet, Austria). Cada muestra fue analizada por triplicado. Los parámetros hematológicos evaluados incluyeron hematocrito (PCV), recuentos de plaquetas (PLT), leucocitos (WBC), valores relativos y absolutos de linfocitos (LYM), monocitos (MID), neutrófilos, eosinófilos y basófilos (GRA).

\section{ANÁLISIS ESTADÍSTICO}

Las variables hematológicas estudiadas presentaron distribución normal (prueba de Kolmogorov-Smirnov $(\mathrm{P}>0,05))$. Los valores de sangre entera y del APC fueron comparados mediante la prueba de Student $(\mathrm{t})$ para muestras pareadas. Los factores sexo y edad fueron analizados mediante un análisis de varianza (ANOVA). Todos los resultados se presentaron como media y error estándar. Se empleó el coeficiente de correlación de Pearson $(\rho)$ para determinar el grado de asociación entre los parámetros evaluados en el APC y en sangre entera. Finalmente, para estudiar la relación entre variables se realizó un análisis de regresión lineal simple. Se aceptó una diferencia estadísticamente significativa de $\mathrm{P} \leq 0,01$ para $t, y \mathrm{P} \leq 0,01$ y $\mathrm{P} \leq 0,05$ para $\rho$. La eficiencia de colección de plaquetas se determinó mediante la fórmula de Weibrich y col (2003): (volumen de APC x recuento de plaquetas en el APC/volumen de sangre entera $\mathrm{x}$ recuento de plaquetas en sangre entera) x 100 .

\section{RESULTADOS Y DISCUSIÓN}

Los recuentos celulares de la sangre entera y de los APCs, presentaron diferencias estadísticamente significativas $(\mathrm{P}<0,01)$ respecto a los valores de PLT, WBC, valores relativos y absolutos de GRA, absolutos de MID, relativos de PCV y LYM, mas no en los valores absolutos de LYM y relativos de MID (cuadro 1). Tampoco se hallaron diferencias entre el sexo y la edad de los animales

Cuadro 1. Valores promedios ( \pm error estándar) de las variables hematológicas en muestras de sangre entera y concentrado de plaquetas.

Mean ( \pm standard error) values for the hematologic variables in the whole blood samples and the platelet concentrates.

\begin{tabular}{|c|c|c|}
\hline Variable & Sangre entera & Concentrado de Plaquetas \\
\hline PLT (fragmentos $\times 10^{3} / \mu \mathrm{L}$ ) & $345,3(44,93)^{*}$ & $515,9 \quad(60,74)$ \\
\hline PCV \% & $44,2(1,96)^{*}$ & $3,97 \quad(1,14)$ \\
\hline WBC (células x $10^{3} / \mu \mathrm{L}$ ) & $16,6(2,08)^{*}$ & $6,9 \quad(0,48)$ \\
\hline LYM (células x $10^{3} / \mu \mathrm{L}$ ) & $5,05(1,45)$ & $3,07 \quad(0,27)$ \\
\hline LYM\% & $26,6(4,01)^{*}$ & $46,6 \quad(5,76)$ \\
\hline MID (células x $10^{3} / \mu \mathrm{L}$ ) & $0,79(0,22)^{*}$ & $0,275(0,04)$ \\
\hline MID \% & $4,2 \quad(0,58)$ & $3,9 \quad(0,50)$ \\
\hline GRA (células $\times 10^{3} / \mu \mathrm{L}$ ) & $10,7(0,93)^{*}$ & $3,6 \quad(0,54)$ \\
\hline GRA\% & $69,3(4,19)^{*}$ & $49,2 \quad(5,53)$ \\
\hline
\end{tabular}

PLT: plaquetas; PCV: hematocrito; WBC: leucocitos; LYM: linfocitos; MID: monocitos; GRA: neutrófilos, eosinófilos y basófilos.

*Variable significativamente diferente $(\mathrm{P}<0,01)$ entre la sangre entera y el concentrado autólogo de plaquetas. 
Cuadro 2. Coeficientes de correlación de Pearson $(\rho)$ y ecuaciones de regresión lineal para la variable dependiente (número de plaquetas en el APC).

Pearson correlation coefficient $(\rho)$ and lineal regression equation for the response variable (numbers of platelets in APC).

\begin{tabular}{lcclc}
\hline Variable & $\rho$ & $\mathrm{P}$ & Ecuación de regresión lineal & $\mathrm{R}^{2}$ \\
\hline PLT & $0,946^{* *}$ & 0,000 & PLT-APC $=74.432,699+1,279$ (PLT) & 0,946 \\
MID & $0,819^{* *}$ & 0,001 & PLT-APC $=337.459,058+225,286$ (MID) & 0,671 \\
GRA & $0,797^{* *}$ & 0,002 & PLT-APC $=41.894,228+51,673$ (GRA) & 0,635 \\
LYM-APC & $-0,702^{*}$ & 0,011 & PLT-APC $=986.142,041-153,162$ (LYM-APC) & 0,493 \\
WBC & $0,602^{*}$ & 0,038 & PLT-APC $=223.619,431+17,572$ (WBC) & 0,363 \\
\hline
\end{tabular}

PLT: plaquetas; PCV: hematocrito; WBC: leucocitos; LYM: linfocitos; MID: monocitos; GRA: neutrófilos, eosinófilos y basófilos.

PLT-APC, número de plaquetas en el concentrado de plaquetas. $* \mathrm{P}<0,05 . * * \mathrm{P}<0,01$.

PLT-APC, numbers of platelets in autologous platelet concentrate. $* \mathrm{P}<0.05$. $* * \mathrm{P}<0.01$.

y los recuentos celulares en el análisis hematológico. Se encontró correlación negativa $(\rho=-0,702 \mathrm{P}=0,01)$ entre el recuento de linfocitos y de plaquetas presentes en los APCs. Las respectivas ecuaciones de regresión lineal son presentadas en el cuadro 2. La eficiencia en la colección de plaquetas fue de $29,9 \%$. La concentración de plaquetas fue 49,4\% superior en los APCs respecto a las muestras de sangre entera.

Jensen y col (2004) describieron un método manual para concentrar plaquetas de perros con EDTA, mediante centrifugación única. Ellos obtenían un incremento de plaquetas y leucocitos en el APC en relación a sangre entera de $670 \%(1.884 .000 \mathrm{PLT} / \mu \mathrm{L})$ y $720 \%(64.200$ $\mathrm{WBC} / \mu \mathrm{L})$, respectivamente. Sin embargo, el EDTA no es un anticoagulante ideal para concentrar plaquetas (White y Escolar 2000). Comparado a los resultados de Jensen y col (2004), el método descrito en este artículo concentra una menor cantidad de plaquetas, mientras que presenta una concentración de leucocitos $58 \%$ inferior. Por otra parte, el uso de APCs con EDTA podría provocar lesiones estructurales, bioquímicas y funcionales a las plaquetas (White y Escolar 2000) y ser irritante para los tejidos tratados.

Existen algunos reportes en los que se describe el uso del citrato de sodio como anticoagulante (Ferraz y col 2007, Bonomi y col 2007, Choi y col 2005, You y col 2007) para concentrar plaquetas caninas. En esos trabajos describen la concentración de plaquetas en esa especie mediante dos tiempos de centrifugación. Normalmente, el producto final de esos protocolos osciló entre 400.000 a 1.300.000 PLT/ $\mu \mathrm{L}$. En esas investigaciones las plaquetas fueron contadas manualmente (microscopia óptica) y no se presentó otra información hematológica adicional de las características de los APCs obtenidos.

El método del tubo planteado en este estudio con una centrifugación única concentra plaquetas de una manera cercana al rango descrito para dos centrifugaciones. Lei y col (2009) concluyeron que la calidad del APC está estrechamente relacionada con el anticoagulante empleado. El uso de ACD produce superior calidad del
APC que cuando se emplea citrato de sodio o heparina. Lo anterior plantea una ventaja del método descrito en este artículo. Li y col (2009) usaron ACD como anticoagulante y mediante doble centrifugación concentraron una media de 1.100.000 PLT/ $\mu \mathrm{L}$ en el APC. Esto corresponde, aproximadamente, al doble de la cantidad de plaquetas concentradas con el método desarrollado por nuestro grupo. Sin embargo, desde el punto de vista clínico y tal como ha sido evaluado en caballos, no existe correlación alguna entre el número de plaquetas concentradas y la respuesta clínica de los caballos tratados (Carmona y col 2011).

La cantidad de plaquetas que se concentran con el método evaluado en este estudio podría ser suficiente para producir efectos biológicos (Weibrich y col 2004, Carmona y col 2011). Es necesario recordar que lo más importante de un método para concentrar plaquetas es que además de concentrar altos números de las mismas permita obtener plaquetas vivas e inactivas (Marx 2004, Carmona y col 2011). El método evaluado permitió concentrar plaquetas y disminuir de manera significativa los MID y GRA y porcentualmente los LYM. Es decir, la población celular concentrada fue en su totalidad diferente a la sanguínea basal. Quizás esa modificación pueda tener alguna influencia en la utilización clínica de los APCs, debido al importante efecto regulador que tienen los neutrófilos, macrófagos y linfocitos sobre la reparación tisular (Park y col 2004). Cabe aclarar que hasta el momento el papel (perjudicial o benéfico) de los WBC no ha sido completamente esclarecido (Carmona y col 2011).

Las correlaciones positivas encontradas entre la concentración de plaquetas en el APC con MID, GRA y cuya significancia tiende a disminuir con la totalidad de los WBC, podrían deberse a la baja velocidad y tiempo inherentes a la técnica de centrifugación y no necesariamente a que la concentración de PLT-APC esté asociada con los valores basales de estas variables. La correlación negativa encontrada entre PLT y LYM en el APC sugiere que a medida que aumenta la cantidad de LYM la concentración de PLT-APC disminuye exponencialmente, 
lo que podría indicar que el método concentra plaquetas asociadas a valores de LYM similares a los encontrados en sangre entera. La correlación altamente significativa entre las concentraciones basales de plaquetas y PLT-APC y la correspondiente ecuación de regresión lineal indican que para este método el pronóstico del valor de PLT-APC estaría basado en un incremento constante de 74.432,699 más 1,279 veces el valor del número de plaquetas en sangre entera. Si bien la eficiencia de recolección de plaquetas se presenta aparentemente baja, esto podría deberse al hecho de que para efectos del presente estudio sólo se obtuvo $1 \mathrm{~mL}$ de plasma por tubo.

Los resultados obtenidos permiten concluir que la metodología empleada para concentrar plaquetas podría ser aceptable para obtener efectos biológicos en la utilización clínica de los APCs como terapia regenerativa en medicina y cirugía canina. El análisis de regresión linear podría permitir predecir el valor de PLT-APC a partir del conocimiento de las variables conocidas en sangre entera tales como PLT, MID, GRA, LYM y WBC. La relevancia del método descrito radica en que permite concentrar plaquetas mediante utilización de ACD como anticoagulante para incrementar la calidad de APC y que éste se obtiene mediante una única centrifugación a baja velocidad y corto tiempo. En el futuro se deberán evaluar las características bioquímicas y ultraestructurales del APC activado, para determinar su calidad biológica como biofármaco autólogo.

\section{RESUMEN}

El objetivo de este estudio fue evaluar un método manual para concentrar plaquetas caninas y en consecuencia producir concentrados autólogos de plaquetas (APCs) para propósitos clínicos y experimentales. Los APCs fueron obtenidos a partir de sangre venosa depositada en tubos con solución ACD que fueron centrifugados a $191 \mathrm{~g}$ durante 6 minutos. Para los recuentos celulares de sangre entera y APCs, se encontraron diferencias estadísticamente significativas $(\mathrm{P}<0,01)$ entre los recuentos de las plaquetas, leucocitos, granulocitos, monocitos, pero no entre los valores de linfocitos. Se encontró una correlación negativa $(\rho=-0,702$ $\mathrm{P}=0,011$ ) entre el número de linfocitos y plaquetas en los APCs. La eficiencia de recolección de plaquetas fue de $29,9 \%$ y la concentración de plaquetas fue de 49,4\% mayor en los APCs, en comparación con las muestras de sangre entera. Los resultados indicaron que este método de centrifugación simple permite concentrar plaquetas caninas.

\section{REFERENCIAS}

Anitua E, I Andia, B Ardanza, P Nurden, AT Nurden. 2004. Autologous platelets as a source of proteins for healing and tissue regeneration. Thromb Haemost 91, 4-15.

Anitua E, M Sanchez, G Orive, I Andia. 2007. The potential impact of the preparation rich in growth factors (PRGF) in different medical fields. Biomaterials 28, 4551-4560.

Argüelles D, JU Carmona, J Pastor, A Iborra, L Viñals, P Martínes, E Bach, M Prades. 2006. Evaluation of single and double centrifugation tube methods for concentrating equine platelets. Res Vet Sci 81, 237-245.

Bonomi S, CR Auada, F Almeida, DC Branco, KB Braga, C Muramoto, M Amaku. 2007. Plasma rico em plaquetas combinado a hidroxiapatita na formação do calo ósseo em fraturas induzidas experimentalmente no rádio de cães. Ciênc Rural 37, 1045-1051.
Carmona JU, M Prades. 2009. Platelet concentrates to treat musculoskeletal disease in horses. VDM Verlag Dr. Müller Aktiengesellschaft \& Co. KG, Saarbrücken, Germany.

Carmona JU, M Prades, D Argüelles. 2009ª . Autologous platelet concentrates as a treatment for soft tissue musculoskeletal lesions in horses. Arch Med Vet 41, 77-82.

Carmona JU, C López, M Prades. 2009 . Use of autologous platelet concentrates obtained by the tube method as a treatment for arthropathies in horses. Arch Med Vet 41, 175-179.

Carmona JU, C López, CE Giraldo. 2011. Uso de concentrados autólogos de plaquetas como terapia regenerativa de enfermedades crónicas del aparato musculoesquelético. Arch Med Vet 43 (1-10).

Casati MZ, BC Vasconcelos-Gurgel, PF Gonçalves, SP Pimentel, G Rochanogueira, JRFH Nociti, EA Sallum. 2007. Platelet-rich plasma does not improve bone regeneration around peri-implant bone defects - A pilot study in dogs. Int J Oral Macillofac Surg 36, 132-136.

Choi BH, SJ Zhu, BY Kim, SH Huh, SH Lee, JH Jung. 2005. Effect of platelet-rich plasma (PRP) concentration on the viability and proliferation of alveolar bone cells: an in vitro study. Int J Oral Maxillofac Surg 34, 420-424.

Dutra CEA, MM Pereira, R Serakides, MCF Rezende. 2008. In vivo evaluation of bioactive glass foams associated with platelet-rich plasma in bone defects. J Tissue Eng Regen Med 2, 221-227.

Ferraz VCM, CRA Ferrigno, A Schmaedecke. 2007. Platelet concentration of plateletrich plasma from dog, obtained through three centrifugation speeds. Braz J Vet Res Anim Sci 44, 435-440.

Jensen TB, O Rahbek, S Overgaard, K Søballe. 2004. Platelet rich plasma and fresh frozen bone allograft as enhancement of implant fixation - An experimental study in dogs. J Orthop Res 22, 653-658.

Lei H, L Gui, R Xiao. 2009. The effect of anticoagulants on the quality and biological efficacy of platelet-rich plasma. Clin Biochem 42, 1452-1460.

Li NY, RT Yuan, T Chen, LQ Chen, XM Jin. 2009. Effect of PlateletRich Plasma and Latissimus Dorsi Muscle Flap on Osteogenesis and Vascularization of Tissue-Engineered Bone in Dogs. J Oral Maxillofac Surg 67,1850-1858.

Marx RE. 2004. Platelet-rich plasma: evidence to support its usage. J Oral Maxillofac Surg 62, 489-496.

Monteiro SO, O M Lepage, CL Theoret. 2009. Effects of platelet-rich plasma on the repair of wounds on the distal aspect of the forelimb in horses. Am J Vet Res 70, 277-282.

Moreno L, J Marín, F Enríquez, G González, L Moreno, L Cisneros, LM Sancha. 2004. Utilización de plasma rico en plaquetas para regeneración periodontal en un perro. Rev Odont Mex 8, 64-69.

Park JE, A Barbul. 2004. Understanding the role of immune regulation in wound healing. Am J Surg 187, 11S-16S.

Thoesen MS, WS Vander- Berg-Foels, T Stokol, KM Rassnick, MS Jacobson, SB Kevi, RJ Todhunter. 2006. Used of a centrifugationbased, point-of-care device for production of canine autologous bone marrow and platelet concentrates. Am J Vet Res 67, 1655-1661.

Thor A, V Franke-Stenport, CB Johansson, L Rasmusson. 2007. Early bone formation in humans bone grafts treated with platelet-rich plasma: preliminary histomorphometric results. Int J Oral Maxillofac Surg 36, 1164-1171.

Weibrich G, KGK Wilfried, B Rainer, WE Hitzler, G Hafner. 2003. The Harvest Smart PRePTM system versus the Friadent-Schütze platelet-rich plasma kit. Clin Oral Impl Res 14, 233-239.

Weibrich G, T Hansen, W Kleis, R Buch, WE Hitzler. 2004. Effect of platelet concentration in platelet-rich plasma on peri-implant bone regeneration. Bone 34, 665-671.

White G, G Escolar. 2000. EDTA-induced changes in platelet structure and function: adhesion and spreading. Platelets 11, 56-61.

You TM, BH Choi, J Li, JH Jung, HJ Lee, SH Lee, SM Jeong. 2007. The effect of platelet-rich plasma on bone healing around implants placed in bone defects treated With Bio-oss: a pilot study in the dog tibia. Oral Surg Oral Med Oral Pathol Oral Radiol Endod 103, 8-12. 